\title{
Epigenetic Inheritance of Centromeres
}

\author{
S. HENIKOFF AND T. FuruYAMA \\ Howard Hughes Medical Institute, Fred Hutchinson \\ Cancer Research Center, Seattle, Washington 98109 \\ Correspondence: steveh@fhcrc.org
}

\begin{abstract}
Centromeres of higher eukaryotes are epigenetically maintained; however, the mechanism that underlies centromere inheritance is unknown. Centromere identity and inheritance require the assembly of nucleosomes containing the CenH3 histone variant in place of canonical H3. Work from our laboratory has led to the proposal that epigenetic inheritance of centromeres evolved as adaptations of $\mathrm{CenH} 3$ and other centromere proteins to resist drive of selfish centromeres during female meiosis. Our molecular studies have revealed that the Drosophila $\mathrm{CenH} 3$ nucleosome is equivalent to half of the canonical $\mathrm{H} 3$ nucleosome and induces positive supercoils, as opposed to the negative supercoils induced by an $\mathrm{H} 3$ nucleosome. CenH3 likewise induces positive supercoils in functional yeast centromeres in vivo. The right-handed wrapping of DNA around the histone core implied by positive supercoiling indicates that centromeric nucleosomes are unlikely to be octameric and that the exposed surfaces holding the nucleosome together would be available for kinetochore protein recruitment. The mutual incompatibility of nucleosomes with opposite topologies could explain how centromeres are efficiently maintained as unique loci on chromosomes. We propose that the opposite wrapping of DNA around a half-nucleosome core particle facilitates a mode of inheritance that does not depend on DNA sequence, DNA modification or protein conformation.
\end{abstract}

Centromeres, the chromosomal sites of attachment to the mitotic spindle, have been familiar to cell biologists for well over a century (Fig. 1). Centromeres were evident to Walther Flemming in his landmark book published in 1882 (Flemming 1882), where he described in detail the processes that result in a single nucleus dividing into two daughter nuclei. Flemming coined the term "chromatin" to describe the deeply staining bodies that were later called "chromosomes" and the term "mitosis" to describe the dynamic process whereby chromosomes split into two. The work of Flemming and his contemporaries provided the groundwork for the immediate acceptance of Mendel's laws on their rediscovery in 1900, and thus the field of genetics was established with the understanding that genetic inheritance is embodied in chromosomes. It therefore might come as a surprise that although the segregation function of centromeres was understood by Flemming, the genetic identity of centromeres and how they are inherited remains a subject of controversy to this day.

Centromeres are defining features of eukaryotic chromosomes and are essential for mitosis, leading to the expectation that they would be specified by DNA sequence. This expectation was initially fulfilled by the discovery of precise correspondences between genetic and molecular maps of centromeres in the budding yeast Saccharomyces cerevisiae, where each of its 16 centromeres occupies an $\sim 120$-bp sequence (Bloom and Carbon 1982). However, subsequent work failed to identify a counterpart of genetic centromeres in other organisms, not only in animals and plants, but also in other fungi. For example, the budding yeast Candida albicans has centromeres that lack any common sequence elements (Baum et al. 2006). That C. albicans centromeres are inherited epigenetically, i.e., independently of DNA se- quence, is indicated by the fact that "neocentromeres" can be made to form at arbitrary sites in the genome (Ketel et al. 2009). Similarly, rare examples of neocentromeres arise spontaneously in human genomes--these are functionally normal, sometimes persisting through multiple human generations without any detectable phenotypic defect (Warburton 2004). Almost 100 human neocentromeres have been identified to date, and as far as can be determined, they arise at unrelated locations in the genome. Sites of human neocentromere formation show no resemblance to the 171-bp $\alpha$ satellite repeat arrays in which all of our native centromeres are embedded and, except for a weak A + T-rich bias, have no sequence motifs in common. Although centromeres in the large majority of eukaryotes lie within highly repetitive alphoid-like satellites, in rice and chickens some centromeres almost entirely lack satellite repeats (Nagaki et al. 2004; Shang et al. 2010). Like human neocentromeres, these native centromeres show no evidence of sequence preference (Yan et al. 2008). Even satellite-rich natural centromeres may be inactivated and reactivated (Han et al. 2009), analogous to the epigenetic silencing of genes. Thus, although centromeres are commonly located within DNA satellites, there do not appear to be DNA sequence requirements for centromere formation in most eukaryotes.

If it is not DNA sequence, then what marks the locations of centromeres and neocentromeres and specifies their attachment to the spindle at mitosis and meiosis, essentially indefinitely? During the past decade, a consensus has emerged in the centromere field that a specialized nucleosome defined by the presence of a distinct histone 3 variant (CenH3; CENP-A in mammals) (Earnshaw and Rothfield 1985; Palmer et al. 1987) forms the obligatory foundation of all centromeres (Bernad et al. 2009). CenH3 nucleo- 

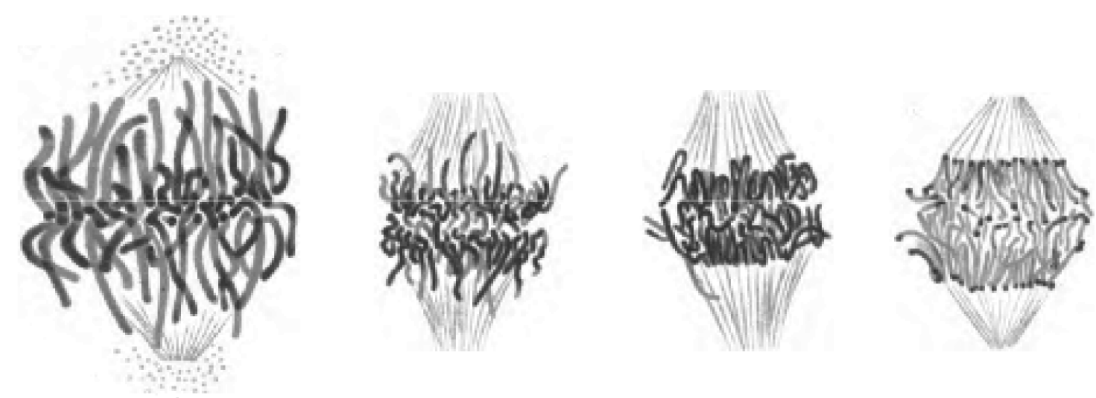

Figure 1. Mitosis in salamander cells as depicted in drawings by Walther Flemming (1882). Note the evident attachment of mitotic spindle fibers to darkly staining regions, later termed "heterochromatin," present on each chromosome. The resulting segregation to the poles indicated to Flemming that there is a single position present on every chromosome that is responsible for anaphase movement of that chromosome to the poles. Flemming called the chromosomes "chromatin" and the process "mitosis."

somes comprise the most fundamental unit of centromere identity, regardless of whether these centromeres are genetic, as in $S$. cerevisiae, are epigenetic, as in most other eukaryotes, or are neocentromeres. $\mathrm{CenH} 3$ nucleosomes are essential for mitosis in almost all organisms studied and at least in Drosophila are both necessary and sufficient for organizing the kinetochore (Heun et al. 2006), the complex proteinaceous structure that attaches to spindle microtubules. Therefore, the question of how centromeres are epigenetically inherited becomes a question of how $\mathrm{CenH} 3$ nucleosomes are assembled and propagated.

In this chapter, we first address the question of why nearly all eukaryotes have evolved an epigenetic mode of centromere inheritance. We then briefly outline other modes of epigenetic inheritance in eukaryotes as background for considering how CenH3 nucleosomes might be inherited. This is followed by a review of our studies of CenH3 nucleosome assembly and on biochemical features of $\mathrm{CenH} 3$ nucleosomes. Finally, we describe our more recent work which shows that $\mathrm{CenH} 3$ nucleosomes are fundamentally different from canonical nucleosomes, in that they wrap DNA in a right-handed, rather than a lefthanded, orientation. This discovery leads us to propose a novel mode of epigenetic inheritance that does not rely on either covalent modification of DNA or protein or protein inheritance per se, but rather on DNA topology.

\section{WHY ARE MOST CENTROMERES EPIGENETIC?}

The astonishing variety of DNA sequences that harbor centromeres and neocentromeres, even within the same organism, presents a paradox (Henikoff et al. 2001). Loss of a single centromere means loss of the chromosome and dominant lethality, and such strong selection should have led to selection for an optimal centromeric sequence. Yet centromeric DNA satellites are among the most rapidly evolving sequences in a genome. We were led to a possible resolution of this paradox with the discovery that $\mathrm{CenH} 3 \mathrm{~s}$ in Drosophila are evolving under positive selection (Malik and Henikoff 2001), and this finding was subsequently extended to CENP-C, a large centromere-specific DNA-binding protein (Talbert et al. 2004) and to other eukaryotes with rapidly evolving centromeres (Malik and Henikoff 2009;
Schueler et al. 2010). Recurrent positive selection implies genetic conflict, and there is one situation, female meiosis, in which centromeres would be expected to compete against one another. That is because only one of the four products of female meiosis is included in the egg nucleus, whereas the other three products are lost as polar bodies. This creates a win-or-lose situation for chromosomes, and thus a centromere that achieves a favorable position relative to the egg pole is more likely to survive to the next generation (Fig. 2 ). A centromere might achieve an advantage simply by satellite expansion to make a larger $\mathrm{CenH} 3$ domain, al-

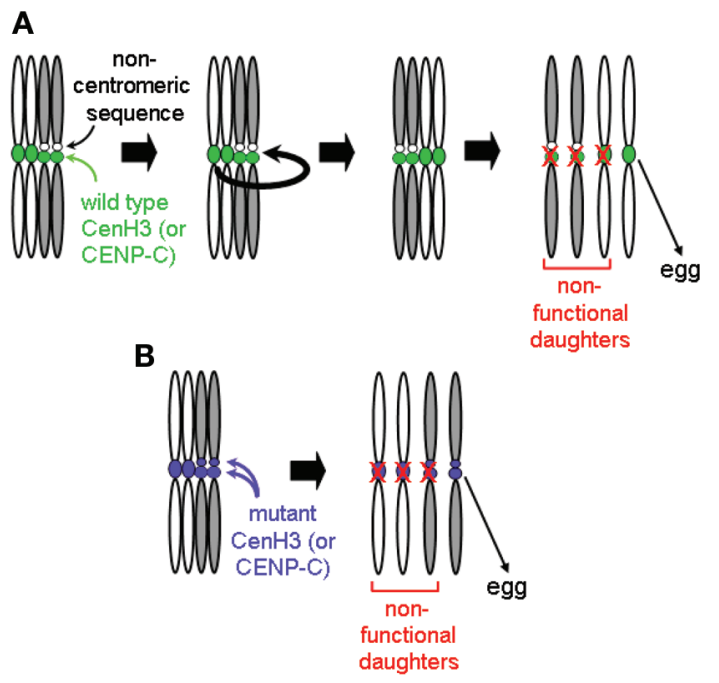

Figure 2. Model for centromere drive during female meiosis. (A) An expanding centromere drives to high frequencies in the population by accumulating more foundation proteins such as $\mathrm{CenH} 3$ and CENP-C (green) and making more microtubule connections, causing it to orient toward the egg pole (curved arrow) even when it starts out in an unfavorable position. Such biased orientation during male meiosis might trigger a checkpoint and result in sterility, which would limit drive. $(B)$ Normal segregation would be restored if the driving centromere encounters a mutant CENP-C (blue) with reduced DNA-binding specificity that binds to previously noncentromeric sequence. Meiotic parity results, providing an advantage for the gene encoding the mutant foundation protein, which sweeps to fixation. (Adapted, with permission, from Dawe and Henikoff 2006 [CElsevier].) 
though the mechanistic basis for preferential orientation on the meiotic spindle is unknown.

Centromere meiotic drive can account for the well-established advantage of human Robertsonian translocations (centromeric fusions of two acrocentric chromosomes to yield a metacentric) in female but not in male meiosis (Daniel 2002) and for anomalous segregation distortion in monkey flower hybrids (Fishman and Saunders 2008). These unusual situations make it possible to detect the consequences of centromere competition; however, we expect that competition between maternally and paternally derived centromeres occurs during every female meiosis but without consequence except to favor one chromosome more often than another. Centromere drive can also be harmful, in that a successfully driving centromere with a deleterious mutation can reduce the fitness of a population (Malik 2009). In addition, centromere drive seems to result in male meiotic defects, likely resulting from misorientation on the meiotic spindle and checkpoint arrest (McKee et al. 1998). For example, although human Robersonian translocations are preferentially transmitted during female meiosis, they cause low fertility of male carriers of this fusion chromosome (Daniel 2002). Therefore, host genomes will have evolved mechanisms to combat centromere drive, and we suppose that CenH3 and CENP-C, which directly interact with centromeric sequences, have been evolving adaptively to neutralize centromere drive and thus to help the host genome achieve parity among competing homologous centromeres.

Avoidance of deleterious centromere drive might have resulted in the evolution of epigenetic centromeres in plants and animals (Dawe and Henikoff 2006). A mutant centromeric DNA satellite sequence that expands and drives to high frequency in a population will eventually encounter a mutant centromere protein that has reduced specificity such that it binds to an existing satellite adjacent to a wild-type centromere (Fig. 2). Restoring balance between the mutant and wild-type homologous centromeres can relieve the male sterility defect, and both the mutant centromere and the gene encoding the mutant centromere protein will drive toward fixation in the population. In this way, the tendency for a selfish centromere to favor its own sequence will be resisted by a centromere protein that reduces sequence specificity. Thus, epigenetic centromeres would have evolved as a successful strategy to resist centromere drive.

Such a process of centromere competition followed by restoration of parity with mutation of a centromere protein represents a Dobzhansky-Muller two-component system, in which two interacting loci evolve together in an isolated population while becoming incompatible with the original population (Presgraves 2010). Crosses between populations would result in renewed drive and deleterious effects on male fertility. In this manner, centromere drive can result in hybrid sterility, providing a molecular explanation for postzygotic reproductive isolation. Recent studies demonstrating that hybrid sterility genes are under positive selection (Oliver et al. 2009; Phadnis and Orr 2009; Presgraves 2010) and that they may involve interactions with centromeric regions (Bayes and Malik 2009) suggest that centromere drive is a common mechanism of speciation. To the extent that speciation leads to successful new forms of life, centromere drive and epigenetic centromeres will persist and thrive.

\section{THREE MODES OF EPIGENETIC INHERITANCE}

Epigenetic inheritance is central to the biology of higher organisms, including developmental biology and stem cell biology. Different cells in an individual inherit the same DNA but have different developmental fates that are propagated through multiple rounds of cell division. One mode of epigenetic inheritance, maintenance DNA methylation, is simple and well understood (Goll and Bestor 2005). DNA methylation occurs primarily on cytosines of CG dinucleotides. If a CG site is methylated on both DNA strands, replication will result in a hemi-methylated site. After encountering such a site, the enzyme Dnmt1 completes the methylation, thus restoring the fully methylated configuration. As long as Dnmt1 methylates only hemimethylated sites, and methylation is irreversible, the methylation pattern will be faithfully inherited from one cell generation to the next. In many eukaryotes, DNA methylation is a mechanism for silencing of parasitic transposable elements and viruses. In addition, the phenomenon of genomic imprinting represents an example of epigenetic inheritance that relies in part on DNA methylation in both plants and animals (Edwards and Ferguson-Smith 2007; Gehring and Henikoff 2007). A gene is imprinted if there is a difference in expression of an allele of a gene depending on whether it was inherited from the mother or the father, and the activity of Dnmt1 is required to stably maintain the silent state of imprinted genes throughout development (Hirasawa et al. 2008).

Other developmental processes do not appear to depend on DNA methylation, and the mechanistic basis for inheritance of gene activity states from the zygote through to the mature adult remains a subject of intense interest. One theory posits that epigenetic inheritance involves a positive-feedback loop (Margueron and Reinberg 2010). An alternative theory is that epigenetic inheritance involves the maintenance of epigenetic marks during replication, by analogy with maintenance DNA methylation (Hansen et al. 2008). A common problem in the literature is that epigenetics is often defined in terms of one or the other theory, a situation that has led to unnecessary confusion (Ptashne 2007). For example, DNA methylation is not maintained by a positive-feedback loop but rather by enzymatic action of the Dnmt1 methyltransferase, which acts in a semiconservative manner to replicate the methylation state of a cytosine. Some popular models for epigenetic inheritance posit that methylation of a histone lysine has a similar role during development (Hansen et al. 2008). We have recently tested whether histone modifications are capable of transmitting an epigenetic state by directly measuring the turnover of nucleosomes genome wide in a Drosophila cell-line system (Deal et al. 2010). We found that sites of epigenetic regulation, including those bound by Polycomb-group proteins, turn over much too rapidly for any histone modification to itself be inherited from one 
cell cycle to the next. This implies that epigenetic inheritance is maintained by a feedback loop, perhaps one that involves nucleosome turnover.

DNA methylation is also a component of a third mode of epigenetic inheritance that is common in bacteria. Dam methyltransferase methylates the adenine bases of nearly all of the $\sim 20,000$ GATC sites present in the Escherichia coli genome; however, it is excluded at some of them because it is blocked by a sequence-specific DNA binding protein (Casadesus and Low 2006). For example, the leucine-response regulatory protein (Lrp) binds to unmethylated GATC sites at the pap operon, thus both blocking methylation by Dam and activating expression of the operon. In this way, mutual exclusion of Dam by Lrp and of Lrp by Dam methylation from pap regulatory sequences maintains either of two stable states of expression.

Below, we ask whether any of these modes of epigenetic inheritance--semiconservative replication as in maintenance DNA methylation, a feedback loop, as in developmental memory, or mutual exclusion, as in Dam/Lrp antagonism--can explain the epigenetic inheritance of centromeres. We find evidence for a novel epigenetic mechanism based on structural differences between nucleosomes with mutually exclusive DNA topology states.

\section{ASSEMBLY OF CENH3 NUCLEOSOMES}

The bulk of nucleosomes is assembled immediately behind the replication fork, although some nucleosomes are deposited outside of replication. Of the four canonical core histones--H2A, H2B, H3, and H4-only H3 is deposited exclusively during replication, and the $\mathrm{H} 3.3$ replacement variant is the exclusive substrate for replication-independent nucleosome assembly on chromosome arms (Ahmad and Henikoff 2002). H3.3 preferentially incorporates at active genes and regulatory elements, and this replacement occurs during histone turnover (Deal et al. 2010). The other sites of conspicuous replication-independent nucleosome assembly are on centromeres, where $\mathrm{CenH} 3$ deposits (Ahmad and Henikoff 2002). Replication-independent assembly of CenH3 nucleosomes is nearly universal (Shelby et al. 2000; Ahmad and Henikoff 2001; Malik and Henikoff 2009), and in Drosophila early embryos, it occurs during anaphase of mitosis (Schuh et al. 2007). The fact that CenH3 nucleosomes do not deposit behind the replication fork would seem to exclude semiconservative replication models for $\mathrm{CenH} 3$ nucleosome inheritance.

Alternatively, $\mathrm{CenH} 3$ nucleosomes might maintain their localization to centromeres via some sort of targeting mechanism (Cleveland et al. 2003; Du et al. 2010). To help understand how $\mathrm{CenH} 3$ nucleosomes become localized to centromeres, we looked for components of the CenH3 preassembly complex that might mediate targeting (Furuyama et al. 2006). Conventional chromatin is maintained in part by histone chaperone complexes. The CAF-1 complex deposits $\mathrm{H} 3$, whereas other complexes, including HirA, deposit H3.3 (Tagami et al. 2004). These soluble complexes had been purified from human cell lines, and we used a similar approach to purify the corresponding complex for CID, the Drosophila CenH3, from S2 cells (Furuyama et al.
2006). We used two different affinity-tag methods for purification and obtained the same result for both. In addition to tagged CID, we found its obligate histone-folding partner $\mathrm{H} 4$ and a single protein chaperone $\mathrm{RbAp} 48$, a highly abundant component of various chromatin assembly, remodeling, and modification complexes. We found that RbAp48 makes direct contact with both H4 and CID in the soluble complex. We also confirmed that this soluble complex is sufficient for nucleosome assembly by showing that RbAp48-CID-H4 reconstituted in vitro is sufficient for chromatin assembly activity, without requiring additional components. The simplicity of the $\mathrm{CenH} 3$ assembly complex contrasts with multisubunit complexes previously described for $\mathrm{H} 3$ and $\mathrm{H} 3.3$. This result implied that $\mathrm{CenH} 3$ is not targeted to centromeres as part of the nucleosome assembly process.

More recent studies have reported the isolation of human CENP-A assembly complexes (Dunleavy et al. 2009; Foltz et al. 2009; Shuaib et al. 2010). Regarding Drosophila, RbAp48 was found to be a stoichiometric component of the soluble complex in two of the studies, and another highly abundant histone chaperone, nucleophosmin, was found in all three studies. A major difference from the Drosophila complex was the presence of a centromere-specific protein, HJURP, which does not exist in flies. HJURP is essential for CENP-A incorporation at centromeres, and in one study, it was shown to facilitate nucleosome assembly (Shuaib et al. 2010). Interestingly, HJURP was shown to be a distant homolog of the yeast CenH3-associated protein Scm3 (Sanchez-Pulido et al. 2009). Therefore, it is possible that CENP-A nucleosome assembly involves some degree of targeting in mammals, perhaps an adaptation to the uniform alphoid character of human centromeres. For instance, a single yeast $\mathrm{CenH} 3$ (Cse4) protein is centered over a sequence that is $\sim 90 \% \mathrm{~A}$ + T (Meluh et al. 1998; Furuyama and Biggins 2007), and $\alpha$ satellite is $\sim 65 \%$ A + T, whereas Drosophila centromeres are embedded in sets of short repeat units with diverse base composition, including AATAT, GAGAA, and CCCGTACTCGGT. Although further studies are needed to determine the roles of HJURP and $\mathrm{Scm} 3$ in localizing $\mathrm{CenH} 3$ to human and yeast centromeres, the lack of any potential targeting component in the Drosophila preassembly complex led us to search for features of CenH3 nucleosomes that might help to account for the apparently untargeted deposition of Drosophila CID.

\section{NATIVE DROSOPHILA CENH3 NUCLEOSOMES ARE "HEMISOMES"}

CenH3s tolerate a high degree of sequence divergence relative to $\mathrm{H} 3 \mathrm{~s}$ and $\mathrm{H} 3.3 \mathrm{~s}$, which are nearly invariant (Henikoff et al. 2001). Even closely related Drosophila species have quite dissimilar amino-terminal tails, and by swap experiments, we had shown that there is a single critical region for CID localization that encompasses Loop 1 of the histonefold domain (Vermaak et al. 2002). By changing each of the critical residues within this region to alanine or glycine, we were able to show that single amino acid residues within this critical region of the histone core are essential for targeting 
to centromeres, as opposed to promiscuous assembly throughout the genome. This observation led us to test the possibility that centromere localization is attributable to some structural features of $\mathrm{CenH} 3$ nucleosomes. We purified endogenous CID nucleosomes from Drosophila S2 cells under physiological ionic conditions and subjected them to a variety of analyses (Dalal et al. 2007b). Classical cross-linking within the native particles indicated that they are tetramers; affinity purification of the particles showed that they contain equimolar amounts of CID, H4, H2A, and $\mathrm{H} 2 \mathrm{~B}$; nuclease digestion experiments showed that they wrap much less DNA than H3 octamers; and electron microscopy (EM) showed that they are beads on a string separated by long linkers and that they resist ionic condensation. Most importantly, atomic force microscopy (AFM) showed that CID nucleosomes are stable particles with half the volume of bulk nucleosomes, which provides completely independent evidence from the cross-linking that $\mathrm{CenH} 3$ nucleosomes are tetrameric. Additional experiments using AFM with recognition imaging on histone core particles released from bulk chromatin confirmed that tetramers are by far the predominant CenH3-containing particle (Fig. 3) (Wang et al. 2008), consistent with a half-nucleosome or "hemisome" structure. The loose packaging of hemisomes suggested a model for the kinetochore in which they are extruded to the surface when chromosomes condense into a quasicrystalline structure at mitosis, making them available to recruit kinetochore structural proteins (Dalal et al. 2007a).

The surprising discovery that native Drosophila $\mathrm{CenH} 3$ nucleosomes are hemisomes stands in contrast to results of in vitro reconstitution studies, in which octameric nucleo-

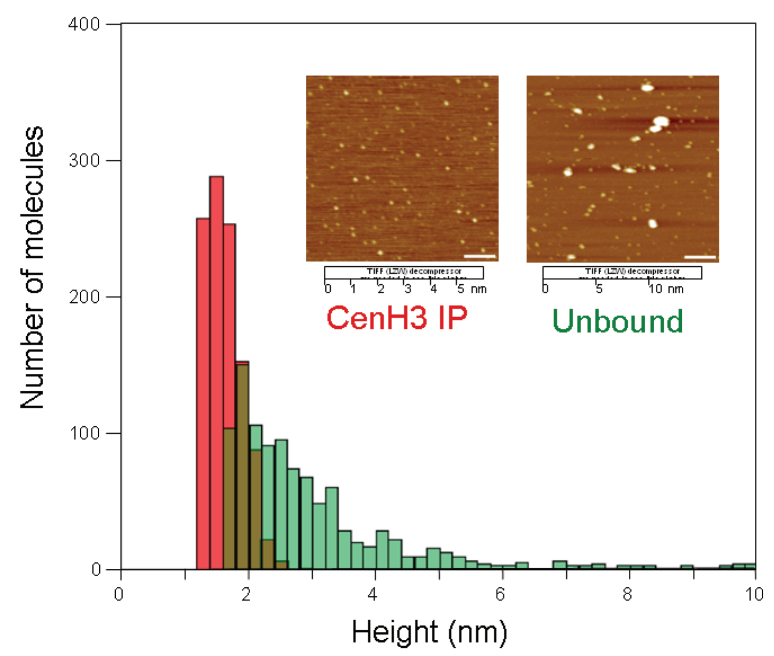

Figure 3. Hydroxylapatite-bound chromatin was purified from interphase cells to enrich for histone core particle complexes immunoprecipitated using an anti-CenH3 antibody and visualized by AFM. The unbound material contains predominantly canonical histone core particles and provided a control. Only particles $>1.65 \mathrm{~nm}$ high and $>6 \mathrm{~nm}$ in radius in the samples were measured to exclude small nonnucleosomal particles and debris. Heights were measured for an equal number of particles for CenH3 (red) and unbound (green) particles. (Insets) Sample images. Bar, 100 $\mathrm{nm}$. The $\mathrm{Z}$ range is shown at the bottom of the inset images. (Reprinted, with permission, from Wang et al. 2008.) somes are readily assembled using dialysis against $2 \mathrm{M} \mathrm{NaCl}$ (150 mM salt is physiological) (Conde e Silva et al. 2007; Camahort et al. 2009; Visnapuu and Greene 2009). Indeed, we found that a variety of large CID-containing particles, including those of octameric size, can be formed during $2 \mathrm{M}$ salt treatment, although we did not detect them in native preparations (Dalal et al. 2007b). Clues from other studies question the in vivo relevance of reconstituted octamers using conditions that have been developed to fold octameric nucleosomes. For example, the dimerization domain of CENP-A octamers is more easily dissociated than that of $\mathrm{H} 3$ octamers, and distinct (CENP-A/H4) 2 tetrasomes fail to form during salt dialysis with DNA, despite the fact that such particles are readily formed using H3 (Conde e Silva et al. 2007). Cse4 octamers could be assembled by salt dialysis onto DNA with the 601 nucleosome-positioning sequence (Lowary and Widom 1998), but these nucleosomes ran as multiple species in native gels, unlike $\mathrm{H} 3$ octameric nucleosomes, which ran as a single species (Camahort et al. 2009). Furthermore, Cse4 octamers reportedly failed to assemble on native yeast centromeric DNA under any tested conditions (Camahort et al. 2009). Direct measurements of native particles isolated under physiological ionic conditions will be needed to ascertain the structure of $\mathrm{Cse} 4$ nucleosomes.

\section{CENH3 NUCLEOSOMES INDUCE POSITIVE DNA SUPERCOILS}

Our studies of CenH3 nucleosome assembly have led to the discovery of another unexpected difference between centromeric and conventional nucleosomes. Using the classical plasmid supercoiling assay for nucleosome assembly, we found that $\mathrm{RbAp} 48$-mediated assembly of reconstituted CenH3 nucleosomes induces positive DNA supercoils in vitro, whereas canonical nucleosomes induce negative supercoils (Furuyama and Henikoff 2009). Using randomly cloned segments of Drosophila DNA, we confirmed that positive supercoiling by reconstituted $\mathrm{CenH} 3$ cores is independent of DNA sequence (Fig. 4). We then extended this observation in vivo, using wild-type and mutant budding yeast minichromosomes maintained in the presence of temperature-sensitive mutations in kinetochore components. Using a series of tandem dicentric minichromosomes, we found that progressive loss of centromeres resulted in progressive loss of positive supercoiling and that positive supercoiling depended on the presence of Cse 4 but not on kinetochore attachment (Fig. 4). We concluded that positive supercoiling by $\mathrm{Cse} 4$ nucleosomes is an intrinsic property of functional yeast centromeres at mitosis.

The most direct interpretation of positive supercoiling induced by CenH3 nucleosomes is that they wrap DNA in a right-handed orientation, the opposite of the left-handed wrapping by $\mathrm{H} 3$ nucleosomes. Alternatively, positive supercoiling might arise by overtwisting of DNA as it wraps around the particle. However, the degree of overtwisting that would be necessary to account for the in vivo topology changes that we measured exceed the plausible limits for B DNA. Moreover, H3 octamers wrap DNA with approximately the same ( $\sim 10 \mathrm{bp} /$ turn $)$ twist as found for B DNA in free solution (Luger et al. 1997), and we have used 
A Drosophila in vitro assembly H3 chromatin CID chromatin

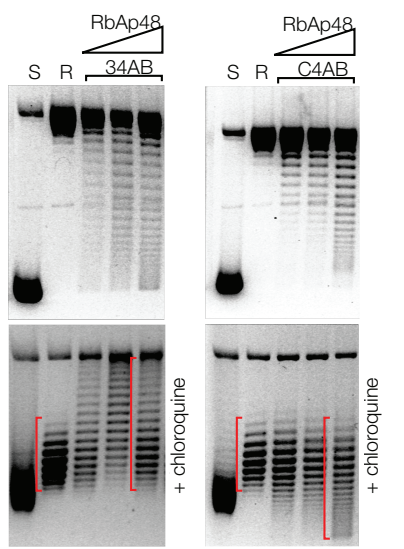

B S. cerevisiae in vivo Cen plasmids

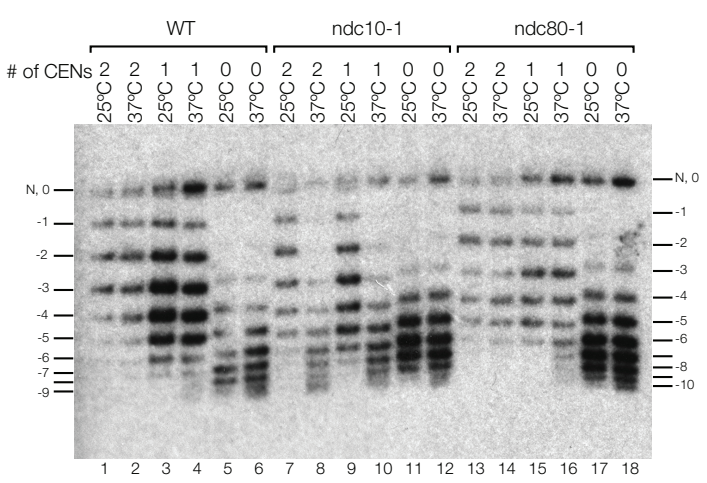

Figure 4. Drosophila CID chromatin assembled in vitro induces positive supercoils in closed circular plasmids. An $\sim 7-\mathrm{kb}$ plasmid containing an 3-kb Drosophila satellite DNA insert (Furuyama et al. 2006) electrophoreses as negatively supercoiled species in an agarose gel after isolation from E. coli (S). The plasmid DNA was relaxed by topoisomerase (R), and H3 (left) or Drosophila CenH3 (CID, right) chromatin was assembled in vitro in the presence of the Drosophila RbAp48 chaperone and histones H4, H2A, and H2B (34AB or $\mathrm{C} 4 \mathrm{AB})$, as previously described. (Top panels) Topoisomer separation on an agarose gel without chloroquine, (bottom panels) topoisomer migration in the presence of $1 \mu \mathrm{g} / \mathrm{mL}$ chloroquine. To visualize DNA, gels were stained with ethidium bromide after separation. The slower migration of topoisomers induced by H3-containing nucleosome relative to the migration of relaxed DNA (R) indicates that $\mathrm{H} 3$ nucleosomes induce negative supercoils (red bracket). The faster migration of topoisomers induced by CID-containing nucleosomes relative to the migration of relaxed DNA $(\mathrm{R})$ in the presence of chloroquine indicates that CID nucleosomes induce positive supercoils in vitro. (B) Cse 4 nucleosomes induce positive supercoils in vivo. Yeast minichromosomes containing 2,1 , or no functional centromeres (\# of CENs) that were derived from wild-type (WT), ndc10-1, or ndc80-1 strains were allowed to go through $\mathrm{S}$ phase at either $25^{\circ} \mathrm{C}$ or $37^{\circ} \mathrm{C}$. Total DNAs were isolated and electrophoresed on an agarose gel containing $0.3 \mu \mathrm{g} / \mathrm{mL}$ chloroquine to resolve topoisomers. Southern blot analysis was performed to detect the minichromosomes. At the restrictive temperature $\left(37^{\circ} \mathrm{C}\right)$, $n d c 10-1$ mutants lose Cse4 from centromeres and lose positive supercoils, whereas $n d c 80-1$ mutants no longer attach centromeres to kinetochore microtubules but, instead, retain positive supercoils. The numbers correspond to the value of net writhe in this chloroquine gel. (N) Nicked, relaxed circles. (Reprinted, with permission, from Furuyama and Henikoff 2009 [CElsevier].)

DNase I mapping to show that reconstituted CID particles also wrap DNA with an 10-bp/turn twist (Furuyama et al. 2006). Thus, the only plausible interpretation of positive supercoiling is that CenH3 nucleosomes wrap DNA in a right-handed orientation, which is opposite to the direction of wrapping found in conventional nucleosomes.

Tetramers of $(\mathrm{H} 3 / \mathrm{H} 4)_{2}$ can wrap DNA in either a left- or right-handed orientation (Hamiche and Richard-Foy 1998), but the addition of two $\mathrm{H} 2 \mathrm{~A} / \mathrm{H} 2 \mathrm{~B}$ dimers results in a lefthanded ramp around which the DNA wraps tightly (Fig. $5 \mathrm{~A}, \mathrm{~B})$. Extensive interaction surfaces between the central tetramer and flanking dimers prevent the nucleosome from springing apart (Luger et al. 1997). These include contacts between the surface formed by the amino-terminal helix of $\mathrm{H} 3$ and the carboxyl terminus of $\mathrm{H} 4$ with the carboxy-terminal docking domain of an $\mathrm{H} 2 \mathrm{~A}$ and a dimerization interaction between the two H2As through their Loop 1 regions (Luger et al. 1997). In a right-handed nucleosome, these interaction surfaces would face away from one another (Fig. $5 C, D)$ and thus, the existence of stable right-handed octamers is unlikely.

The presence of key residues at the $\mathrm{H} 3 / \mathrm{H} 3$ dimerization interface that are invariant in CenH3s suggests that it is sometime occupied. CENP-A is recruited to sites of double-strand breaks without accompanying $\mathrm{H} 2 \mathrm{~A} / \mathrm{H} 2 \mathrm{~B}$ dimers (Zeitlin et al. 2009), and one can envision conservation of the dimerization surface to form homotypic (CENP-A/H4), tetramers that would compete with and evict resident $\mathrm{H}_{3}$ nucleosomes to facilitate repair. Another possibility is that this interface has been retained to permit CenH3/H3 hybrid formation (Foltz et al. 2006), which would result in left/right core particles that should be unable to stably wrap DNA. CenH3 incorporation into chromosome arms occurs under many circumstances (Henikoff et al. 2000; Blower and Karpen 2001; Van Hooser et al. 2001; Talbert et al. 2002; Tomonaga et al. 2003; Cervantes et al. 2006; Lefrancois et al. 2009; Zeitlin et al. 2009) yet is potentially catastrophic, causing dicentric formation, chromosome loss, and dominant lethality (Tomonaga et al. 2003; Heun et al. 2006). By retaining the ability to dimerize with $\mathrm{H} 3$, misincorporated CenH3s would predominantly form structurally defective left/right CenH3/H3 hybrid particles in chromosome arms. Misincorporated $\mathrm{CenH} 3 \mathrm{~s}$ are degraded by proteolysis (Collins et al. 2004; Moreno-Moreno et al. 2006), and we suggest that these are hybrid CenH3/H3 hexamers or lefthanded $\mathrm{CenH} 3 / \mathrm{CenH} 3$ octamers formed from CenH3 misincorporation during conventional nucleosome assembly on chromosome arms. This process would prevent the formation of dicentrics and thus result in retention of the dimerization interface during CenH3 evolution.

\section{CONTROVERSIES SURROUNDING RIGHT-HANDED HEMISOMES}

Whether right-handed hemisomes are the universal form of CenH3 nucleosomes remains an open question (Dechassa 
A H3 octamer

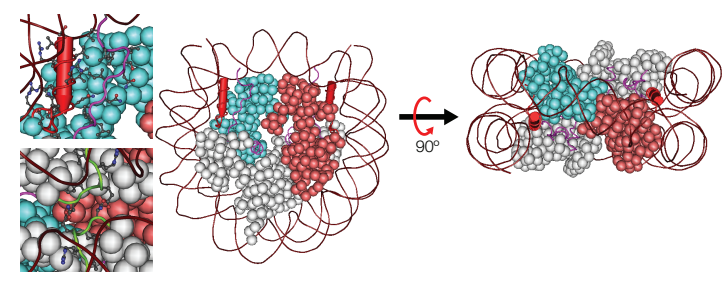

C Left-handed octamer

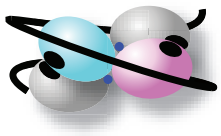

E $1 / 2$ of octamer (left-handed)

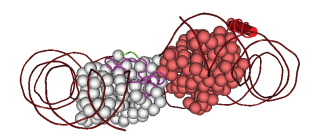

D Right-handed octamer

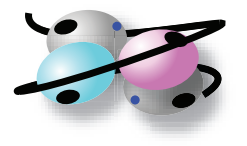

F CenH3 hemisome (right-handed)

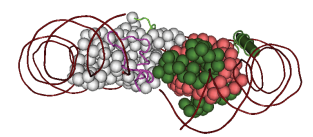

Figure 5. Structures and model of a right-handed hemisome. (A) Crystal structure of a nucleosome (Luger et al. 1997). One H3/H4 dimer is shown in light blue, the second $\mathrm{H} 3 / \mathrm{H} 4$ dimer in light red, both $\mathrm{H} 2 \mathrm{~A} / \mathrm{H} 2 \mathrm{~B}$ dimers are shown in gray, and DNA is shown in brown wrapping around the histone octamer. Close-up view of the amino-terminal helix of $\mathrm{H} 3$ (red stick) and $\mathrm{H} 2 \mathrm{~A}$ docking domain (magenta) with their side chains shown (top inset). (Bottom inset) Interaction between two $\mathrm{H} 2 \mathrm{~A}$ molecules at the bottom of the octameric structure through their Loop 1 (green). (B) Structure in $A$ rotated $90^{\circ}$ to emphasize the spiral of four histone dimers wrapping DNA in the left-hand orientation. $(C)$ and $(D)$ Images of left-handed and putative right-handed octamers. The two $\mathrm{H} 3 / \mathrm{H} 4$ dimers were differentially colored (light blue and magenta), and the two $\mathrm{H} 2 \mathrm{~A} / \mathrm{H} 2 \mathrm{~B}$ dimers are shown in gray. The DNA double helix is shown as a black line. Black ovals in $C$ depict the interaction between the amino-terminal helix of $\mathrm{H} 3$ and the docking domain of H2A. (Blue dots ) H2A Loop1. In the right-handed octamer, which does not exist, the indicated interaction surfaces would face away from one another. $(E)$ Half of the left-handed structure shown in $B$, illustrating exposed surfaces in the absence of the other half. $(F)$ Image of how a right-handed structure might look. $(F)$ Produced using Abobe Photoshop, maintaining the approximate orientation of H4/H2B four-helix bundle. (Reprinted, with permission, from Furuyama and Henikoff 2009 [CElsevier].)

et al. 2009), and various arguments have been put forward that have challenged the generality of our results (Mizuguchi et al. 2007; Black and Bassett 2008; Marshall et al. 2008; Camahort et al. 2009; Hill and Williams 2009). One challenge is the ease with which reconstituted $\mathrm{CenH} 3$ octameric nucleosomes can be produced using conditions that were developed for reconstituting $\mathrm{H} 3$ nucleosomes, although as pointed out above, direct in vivo evidence for the existence of octamers is lacking. Another challenge comes from the criticism of using cross-linking as evidence for tetrameric CID nucleosomes, because CID lacks cross-linkable lysines in the dimerization domain (Black and Bassett 2008). However, this argument overlooks the fact that the classical crosslinking reageant that we used, dimethylsuberimidate, was chosen because it has an $11 \AA$ linker and is known to crosslink only H2A, H2B, and H4 lysines far away from one an- other across the solvent-accessible surface of the H3 nucleosome (Kornberg and Thomas 1974; Suda and Iwai 1979). In any case, the lysines in the $\mathrm{H} 3$ dimerization domain are evidently not solvent accessible in the nucleosome core particle (Lambert and Thomas 1986; Davey et al. 2002). This criticism also overlooked the fact that we were indeed able to cross-link CenH3 octamers reconstituted in vitro, although we never observed these forms in native $\mathrm{CenH} 3$ chromatin from nuclei (Dalal et al. 2007b). Imaging of native $\mathrm{CenH} 3$ particles in solution by AFM provided direct evidence that $\mathrm{CenH} 3$ nucleosomes are tetrameric, confirming the most straightforward interpretation of the cross-linking results. Our additional AFM study of CID nucleosomes using recognition imaging provided further direct evidence that they are tetrameric (Wang et al. 2008), and thus far, no other single-molecule determinations of in vivo CenH3 nucleosomal features have appeared that contradict our findings. A Förster resonance energy transfer (FRET) study of CENP-A nucleosomes was interpreted in terms of an octameric nucleosome (Hemmerich et al. 2008), and this interpretation was cited as contradicting the existence of CENP-A hemisomes (Marshall et al. 2008). However, the analysis in question was completed before our study appeared, and we believe that hemisomes are equally consistent with the FRET data.

Another challenge to the existence of right-handed hemisomes at centromeres comes from a study showing that immunoprecipitation of epitope-tagged Drosophila CID nucleosomes led to the recovery of untagged endogenous CID (Erhardt et al. 2008). Although the authors did not explain how they might have obtained such different results from ours, we note that they digested with micrococcal nuclease (MNase) in the standard manner for obtaining mostly mononucleosomes, whereas we used very light MNase digestion, because even slightly higher levels of digestion led to complete loss of hemisome particles (Dalal et al. 2007b). Under the high MNase digestion conditions used in this study, we expect that the only surviving particles will be misincorporated tagged $\mathrm{CenH} 3$ from chromosome arms that might well be present in the form of octamers. In support of this interpretation, we note that when tagged Cse 4 replaces endogenous $\mathrm{Cse} 4$, it incorporates promiscuously throughout the genome and very prominently at promoters of the most highly active genes (Lefrancois et al. 2009). This mapping was performed using Cse4 ChIP-seq (chromatin immunoprecipitation sequence), where the large majority (95\%) of sequence reads were derived from outside of centromeres. This suggests that misincorporation of tagged $\mathrm{CenH} 3$ expressed under the normal Cse4 promoter can be extensive even when the cells grow normally with no evidence of aberrant chromosome segregation. One way to distinguish bona fide $\mathrm{CenH} 3$ chromatin from noncentromeric material is to examine by EM or AFM (Dalal et al. 2007b), because centromeric nucleosomes are found in long arrays (Blower et al. 2002), whereas misincorporated $\mathrm{CenH} 3$ particles should be interspersed with canonical nucleosomes.

Two other challenges to the generality of right-handed hemisomes have appeared since publication of our $\mathrm{CenH} 3$ topology study. Lavelle et al. (2009) accepted our evidence for right-handed DNA topology but argued that our findings might still be consistent with a right-handed tetrasome as 
opposed to a hemisome. They based this model on in vitro evidence that torsional strain induced by RNA polymerase transit through $\mathrm{H} 3$ nucleosomes can cause the left-handed octamer to unwind and to then rewind into an open righthanded "reversome" (Bancaud et al. 2007). Although we do not doubt that this interesting process might sometimes occur in vivo during transcription, we find it difficult to accept this interpretation of our studies. Reversomes are transient structures that require sustained high torsional stress in order to flip a left-handed octamer into a right-handed configuration. Lavelle and coworkers proposed that such an unstable intermediate might become stabilized following loss of $\mathrm{H} 2 \mathrm{~A} / \mathrm{H} 2 \mathrm{~B}$ dimers via unknown protein-protein interactions. However, our ability to induce positive supercoils using purified histones, RbAp48, and relaxed plasmid circles, without the addition of any machinery for generating torsional stress, demonstrates that no such elaborate mechanism is required to obtain $\mathrm{CenH} 3$ nucleosomes that wrap DNA in a right-handed orientation.

A more direct challenge to the existence of right-handed hemisomes at centromeres comes from a study that claimed to demonstrate that yeast $\mathrm{Cse} 4$ nucleosomes are octamers (Camahort et al. 2009). These authors described a variety of in vitro and in vivo experiments that led them to this conclusion, although the use of the 601 sequence for their in vitro studies would strongly favor the formation of octamers, insofar as this sequence was derived by in vitro selection for octameric nucleosomes that are more stable than any found in nature (Lowary and Widom 1998). One line of evidence was their ability to assemble octameric Cse 4 nucleosomes in vitro using dialysis from $2 \mathrm{M}$ $\mathrm{NaCl}$, which we do not consider convincing, as detailed above. A second line of evidence that Cse4 nucleosomes are octamers was that alanine scanning mutagenesis of the entire protein produced only six lethal mutations, five of which lie within the carboxy-terminal portion that encompasses the $\mathrm{H} 3$ dimerization surface that mediates octamer formation. However, as pointed out above, there are alternative explanations for maintenance of this dimerization surface, and in any case, all six of these sites are among the 10 sites at which both $\mathrm{H} 3 \mathrm{~s}$ and $\mathrm{CenH} 3 \mathrm{~s}$ have the same invariant amino acid (Talbert et al. 2008). A more parsimonious interpretation of their mutagenesis data is that the mutations that they recovered are necessary for the folding of all histone 3 variants. Camahort et al. (2009) argued that if these residues are important for dimerization, they should prevent both octamer formation in vitro and interactions in vivo. Although some of the mutations do indeed cause such effects, there was no correlation between the efficiency of a mutant Cse 4 to assemble in vitro and to be recovered in vivo $\left(R^{2}=0.02\right)$.

The results described above are at least as consistent with hemisomes as they are with octamers. However, Camahort and coworkers also described one series of experiments that led them to specifically argue against Cse4 hemisomes. Using two Cse4 constructs labeled with different epitope tags, they showed that immunoprecipitation of one tag resulted in recovery of a portion of the other tag, although abundant noncentromeric incorporation of tagged Cse 4 (Lefrancois et al. 2009), also evident in their experiments
(Camahort et al. 2009), complicates interpretation of this result. Therefore, it is unclear whether the coimmunoprecipitated complexes were derived from centromeres or were nonfunctional particles from elsewhere in the genome. To obtain centromere-specific information, Camahort et al. (2009) used sequential ChIP to show that the tags are in proximity of one another at a yeast centromere that had previously been shown to have only a single $\mathrm{CenH} 3$ nucleosome (Furuyama and Biggins 2007). However, one limitation of this approach is that by using cross-linking and sonication, which yields fragments that span multiple nucleosomes, it is not clear that both tags were on a single nucleosome. In addition, their experiment was not accompanied by a positive control that would reveal whether the material recovered in the second immunoprecipitation was derived from true centromeric nucleosomes or was experimental background. Such a control is crucial, because hemisomes are predicted to yield nothing in the second affinity purification of sequential ChIP. Experimental issues aside, no explanation was provided for how to fit an octamer, which wraps $\sim 150$ bp of DNA, into the confines of a functional centromere that is known to be occupied by two sequence-specific DNA-binding protein complexes: Cbf1, which sits over the conserved 8-bp CDEI motif, and Cbf3, which sits over the conserved 26-bp CDEIII motif. This would seem to leave only the $~ 80$-bp 90\% ATrich CDEII sequence available for wrapping around the Cse 4 nucleosome, which is just enough room to wrap a tetramer.

\section{CONCLUSIONS}

The surprising properties of CenH3 nucleosomes that we have uncovered fit neither the semiconservative replication nor the feedback loop modes of epigenetic inheritance. Semiconservative replication is unlikely, both because $\mathrm{CenH} 3$ is not deposited following replication and templating on a hemisome would yield an octamer, not another hemisome. Furthermore, a self-perpetuating feedback loop does not account for the many cases in which $\mathrm{CenH} 3$ nucleosomes deposit outside of centromeres and yet do not propagate themselves. Rather, we propose that right-handed wrapping of $\mathrm{CenH} 3$ particles, and their presence at centromeres in uncondensed arrays with long linkers (Dalal et al. 2007a), is sufficient to ensure that new CenH3s assemble into nucleosomes only in gaps between resident $\mathrm{CenH} 3 \mathrm{nu}-$ cleosomes. These gaps might be just large enough to accommodate new hemisomes but not octamers. Exclusion of $\mathrm{H} 3$ and H3.3 octamer assembly from the highly unconventional CenH3 chromatin, and incorporation of mislocalized CenH3 into defective hybrid CenH3/H3 and CenH3/H3.3 on chromosome arms where $\mathrm{H} 3$ and $\mathrm{H} 3.3$ assembly machineries dominate, would lead to perpetual maintenance of centromeres as the exclusive sites of CenH3 hemisome assembly. How this mutual exclusion might be accomplished is unknown, but it is attractive to think that the quiescence of centromeric heterochromatin excludes active processes that would otherwise disrupt CenH3 arrays (Yan et al. 2008). The mutual incompatibility of nucleosome cores that wrap DNA around either left-handed octamers or right-handed hemisomes suggests a novel mechanism for the self-perpetuation of centromeric chromatin. 


\section{ACKNOWLEDGMENTS}

We thank Yamini Dalal for her many key contributions to the chromatin studies described in this chapter, to Harmit Malik for spearheading the evolutionary studies, and to other past and present members of our laboratory for contributing to ideas put forward in this chapter. We are supported by the Howard Hughes Medical Institute.

\section{REFERENCES}

Ahmad K, Henikoff S. 2001. Centromeres are specialized replication domains in heterochromatin. J Cell Biol 153: 101-110.

Ahmad K, Henikoff S. 2002. The histone variant H3.3 marks active chromatin by replication-independent nucleosome assembly. Mol. Cell 9: 1191-1200.

Bancaud A, Wagner G, Conde ESN, Lavelle C, Wong H, Mozziconacci J, Barbi M, Sivolob A, Le Cam E, Mouawad L, et al 2007. Nucleosome chiral transition under positive torsional stress in single chromatin fibers. Mol Cell 27: 135-147.

Baum M, Sanyal K, Mishra PK, Thaler N, Carbon J. 2006. Formation of functional centromeric chromatin is specified epigenetically in Candida albicans. Proc Natl Acad Sci 103: 14877-14882.

Bayes JJ, Malik HS. 2009. Altered heterochromatin binding by a hybrid sterility protein in Drosophila sibling species. Science 326: $1538-1541$

Bernad R, Sánchez P, Losada A. 2009. Epigenetic specification of centromeres by CENP-A. Exp Cell Res 315: 3233-3241.

Black BE, Bassett EA. 2008. The histone variant CENP-A and centromere specification. Curr Opin Cell Biol 20: 91-100.

Bloom KS, Carbon J. 1982. Yeast centromere DNA is in a unique and highly ordered structure in chromosomes and small circular minichromosomes. Cell 29: 305-317.

Blower MD, Karpen GH. 2001. The role of Drosophila CID in kinetochore formation, cell-cycle progression and heterochromatin interactions. Nat Cell Biol 3: 730-739.

Blower MD, Sullivan BA, Karpen GH. 2002. Conserved organization of centromeric chromatin in flies and humans. Dev Cell 2: 319-330.

Camahort R, Shivaraju M, Mattingly M, Li B, Nakanishi S, Zhu D, Shilatifard A, Workman JL, Gerton JL. 2009. Cse4 is part of an octameric nucleosome in budding yeast. Mol Cell 35: 794-805.

Casadesus J, Low D. 2006. Epigenetic gene regulation in the bacterial world. Microbiol Mol Biol Rev 70: 830-856.

Cervantes MD, Xi X, Vermaak D, Yao MC, Malik HS. 2006. The CNA1 histone of the ciliate Tetrahymena thermophila is essential for chromosome segregation in the germline micronucleus. Mol Biol Cell 17: 485-497.

Cleveland DW, Mao Y, Sullivan KF. 2003. Centromeres and kinetochores: From epigenetics to mitotic checkpoint signalling. Cell 112: 407-421.

Collins KA, Furuyama S, Biggins S. 2004. Proteolysis contributes to the exclusive centromere localization of the yeast Cse4/CENPA histone H3 variant. Curr Biol 14: 1968-1972.

Conde e Silva N, Black BE, Sivolob A, Filipski J, Cleveland DW, Prunell A. 2007. CENP-A-containing nucleosomes: Easier disassembly versus exclusive centromeric localization. $J$ Mol Biol 370: $555-573$.

Dalal Y, Furuyama T, Vermaak D, Henikoff S. 2007a. Structure, dynamics, and evolution of centromeric nucleosomes. Proc Nat Acad Sci 104: 15974-15981.

Dalal Y, Wang H, Lindsay S, Henikoff S. 2007b. Tetrameric structure of centromeric nucleosomes in Drosophila cells. PLoS Biol 5: e218.

Daniel A. 2002. Distortion of female meiotic segregation and reduced male fertility in human Robertsonian translocations: Consistent with the centromere model of co-evolving centromere DNA/centromeric histone (CENP-A). Am J Med Genet 111: 450-452.

Davey CA, Sargent DF, Luger K, Maeder AW, Richmond TJ. 2002. Solvent mediated interactions in the structure of the nucleosome core particle at $1.9 \AA$ resolution. J Mol Biol 319: 1097-1113.

Dawe RK, Henikoff S. 2006. Centromeres put epigenetics in the driver's seat. Trends Biochem Sci 31: 662-669.

Deal RB, Henikoff JG, Henikoff S. 2010. Genome-wide kinetics of nucleosome turnover determined by metabolic labeling of histones. Science 328: 1161-1164.

Dechassa ML, D'Arcy S, Luger K. 2009. A positive spin on the centromere. Cell 138: 22-24.

Du Y, Topp CN, Dawe RK. 2010. DNA binding of centromere protein C (CENPC) is stabilized by single-stranded RNA. PLoS Genet 6: e1000835.

Dunleavy EM, Roche D, Tagami H, Lacoste N, Ray-Gallet D, Nakamura Y, Daigo Y, Nakatani Y, Almouzni-Pettinotti G. 2009. HJURP is a cell-cycle-dependent maintenance and deposition factor of CENP-A at centromeres. Cell 137: 485-497.

Earnshaw WC, Rothfield N. 1985. Identification of a family of human centromere proteins using autoimmune sera from patients with scleroderma. Chromosoma 91: 313-321.

Edwards CA, Ferguson-Smith AC. 2007. Mechanisms regulating imprinted genes in clusters. Curr Opin Cell Biol 19: 281-289.

Erhardt S, Mellone BG, Betts CM, Zhang W, Karpen GH, Straight AF. 2008. Genome-wide analysis reveals a cell cycle-dependent mechanism controlling centromere propagation. $J$ Cell Biol 183: 805-818.

Fishman L, Saunders A. 2008. Centromere-associated female meiotic drive entails male fitness costs in monkeyflowers. Science 322: $1559-1562$.

Flemming W. 1882. Zellsubstanz, Kern und Zelltheilung. F.C.W. Vogel, Leipzig.

Foltz DR, Jansen LE, Black BE, Bailey AO, Yates JR 3rd, Cleveland DW. 2006. The human CENP-A centromeric nucleosomeassociated complex. Nat Cell Biol 8: 458-469.

Foltz DR, Jansen LE, Bailey AO, Yates JR 3rd, Bassett EA, Wood S, Black BE, Cleveland DW. 2009. Centromere-specific assembly of CENP-a nucleosomes is mediated by HJURP. Cell 137: $472-484$

Furuyama S, Biggins S. 2007. Centromere identity is specified by a single centromeric nucleosome in budding yeast. Proc Natl Acad Sci 104: 14706-14711.

Furuyama T, Henikoff S. 2009. Centromeric nucleosomes induce positive DNA supercoils. Cell 138: 104-113.

Furuyama T, Dalal Y, Henikoff S. 2006. Chaperone-mediated assembly of centromeric chromatin in vitro. Proc Natl Acad Sci 103: 6172-6177.

Gehring M, Henikoff S. 2007. DNA methylation dynamics in plant genomes. Biochim Biophys Acta 1769: 276-286.

Goll MG, Bestor TH. 2005. Eukaryotic cytosine methyltransferases. Аnпи Rev Biochem 74: 481-514.

Hamiche A, Richard-Foy H. 1998. The switch in the helical handedness of the histone $(\mathrm{H} 3-\mathrm{H} 4) 2$ tetramer within a nucleoprotein particle requires a reorientation of the $\mathrm{H} 3-\mathrm{H} 3$ interface. $J$ Biol Chem 273: 9261-9269.

Han F, Gao Z, Birchler JA. 2009. Reactivation of an inactive centromere reveals epigenetic and structural components for centromere specification in maize. Plant Cell 21: 1929-1939.

Hansen KH, Bracken AP, Pasini D, Dietrich N, Gehani SS, Monrad A, Rappsilber J, Lerdrup M, Helin K. 2008. A model for transmission of the H3K27me3 epigenetic mark. Nat Cell Biol 10: $1291-1300$

Hemmerich P, Weidtkamp-Peters S, Hoischen C, Schmiedeberg L, Erliandri I, Diekmann S. 2008. Dynamics of inner kinetochore assembly and maintenance in living cells. J Cell Biol 180: $1101-1114$

Henikoff S, Ahmad K, Platero JS, van Steensel B. 2000. Heterochromatic deposition of centromeric histone H3-like proteins. Proc Natl Acad Sci 97: 716-721.

Henikoff S, Ahmad K, Malik HS. 2001. The centromere paradox: Stable inheritance with rapidly evolving DNA. Science 293: 1098-1102.

Heun P, Erhardt S, Blower MD, Weiss S, Skora AD, Karpen GH. 2006. Mislocalization of the Drosophila centromere-specific histone CID promotes formation of functional ectopic kinetochores. Dev Cell 10: 303-315.

Hill E, Williams R. 2009. Super-coil me: Sizing up centromeric 
nucleosomes. J Cell Biol 186: 453-456.

Hirasawa R, Chiba H, Kaneda M, Tajima S, Li E, Jaenisch R, Sasaki H. 2008. Maternal and zygotic Dnmt1 are necessary and sufficient for the maintenance of DNA methylation imprints during preimplantation development. Genes Dev 22: $1607-$ 1616.

Ketel C, Wang HS, McClellan M, Bouchonville K, Selmecki A, Lahav T, Gerami-Nejad M, Berman J. 2009. Neocentromeres form efficiently at multiple possible loci in Candida albicans. PLoS Genet 5: e1000400.

Kornberg RD, Thomas JO. 1974. Chromatin structure; oligomers of the histones. Science 184: 865-868.

Lambert SF, Thomas JO. 1986. Lysine-containing DNA-binding regions on the surface of the histone octamer in the nucleosome core particle. Eur J Biochem 160: 191-201.

Lavelle C, Recouvreux P, Wong H, Bancaud A, Viovy JL, Prunell A, Victor JL. 2009. Right-handed nucleosome: Myth or reality? Cell 139: 1216-1217.

Lefrancois P, Euskirchen GM, Auerbach RK, Rozowsky J, Gibson T, Yellman CM, Gerstein M, Snyder M. 2009. Efficient yeast ChIP-Seq using multiplex short-read DNA sequencing. BMC Genomics 10: 37.

Lowary PT, Widom J. 1998. New DNA sequence rules for high affinity binding to histone octamer and sequence-directed nucleosome positioning. $J$ Mol Biol 276: 19-42.

Luger K, Mader AW, Richmond RK, Sargent DF, Richmond TJ. 1997. Crystal structure of the nucleosome core particle at 2.8 Å resolution. Nature 389: 251-260.

Malik HS. 2009. The centromere-drive hypothesis: A simple basis for centromere complexity. Prog Mol Subcell Biol 48: 33-52.

Malik HS, Henikoff S. 2001. Adaptive evolution of Cid, a centromere-specific histone in Drosophila. Genetics 157: 12931298.

Malik HS, Henikoff S. 2009. Major evolutionary transitions in centromere complexity. Cell 138: 1067-1082.

Margueron R, Reinberg D. 2010. Chromatin structure and the inheritance of epigenetic information. Nat Rev Genet 11: 285-296.

Marshall OJ, Marshall AT, Choo KH. 2008. Three-dimensional localization of CENP-A suggests a complex higher order structure of centromeric chromatin. J Cell Biol 183: 1193-1202.

McKee BD, Wilhelm K, Merrill C, Ren X. 1998. Male sterility and meiotic drive associated with sex chromosome rearrangements in Drosophila. Role of X-Y pairing. Genetics 149: 143-155.

Meluh PB, Yang P, Glowczewski L, Koshland D, Smith MM. 1998. $\mathrm{Cse} 4 \mathrm{p}$ is a component of the core centromere of Saccharomyces cerevisiae. Cell 94: 607-613.

Mizuguchi G, Xiao H, Wisniewski J, Smith MM, Wu C. 2007. Nonhistone $\mathrm{Scm} 3$ and histones CenH3-H4 assemble the core of centromere-specific nucleosomes. Cell 129: 1153-1164.

Moreno-Moreno O, Torras-Llort M, Azorin F. 2006. Proteolysis restricts localization of CID, the centromere-specific histone $\mathrm{H} 3$ variant of Drosophila, to centromeres. Nucleic Acids Res 34: 6247-6255.

Nagaki K, Cheng Z, Ouyang S, Talbert PB, Kim M, Jones KM, Henikoff S, Buell CR, Jiang J. 2004. Sequencing of a rice centromere uncovers active genes. Nat Genet 36: 138-145.

Oliver PL, Goodstadt L, Bayes JJ, Birtle Z, Roach KC, Phadnis N, Beatson SA, Lunter G, Malik HS, Ponting CP. 2009. Accelerated evolution of the Prdm9 speciation gene across diverse metazoan taxa. PLoS Genet 5: e1000753.

Palmer DK, O'Day K, Wener MH, Andrews BS, Margolis RL. 1987. A $17-\mathrm{kD}$ centromere protein (CENP-A) copurifies with nucleosome core particles and with histones. J Cell Biol 104: 805-815.

Phadnis N, Orr HA. 2009. A single gene causes both male sterility and segregation distortion in Drosophila hybrids. Science 323: 376-379.
Presgraves DC. 2010. The molecular evolutionary basis of species formation. Nat Rev Genet 11: 175-180.

Ptashne M. 2007. On the use of the word 'epigenetic'. Curr Biol 17: R233-R236.

Sanchez-Pulido L, Pidoux AL, Ponting CP, Allshire RC. 2009. Common ancestry of the CENP-A chaperones Scm3 and HJURP. Cell 137: 1173-1174.

Schueler MG, Swanson W, Thomas PJ, NISC Comparative Sequencing Program, Green ED. 2010. Adaptive evolution of foundation kinetochore proteins in primates. Mol Biol Evol 27: $1585-1597$.

Schuh M, Lehner CF, Heidmann S. 2007. Incorporation of Drosophila CID/CENP-A and CENP-C into centromeres during early embryonic anaphase. Curr Biol 17: 237-243.

Shang W-H, Hori T, Toyoda A, Kato J, Popendorf K, Sakakibara Y, Fujiyama A, Fukagawa T. 2010. Chickens possess centromeres with both extended tandem repeats and short non-tandem-repetitive sequences. Genome Res 20: 1219-1228.

Shelby RD, Monier K, Sullivan KF. 2000. Chromatin assembly at kinetochores is uncoupled from DNA replication. J Cell Biol 151: 1113-1118.

Shuaib M, Ouararhni K, Dimitrov S, Hamiche A. 2010. HJURP binds CENP-A via a highly conserved N-terminal domain and mediates its deposition at centromeres. Proc Natl Acad Sci 107: 1349-1354.

Suda M, Iwai K. 1979. Identification of suberimidate cross-linking sites of four histone sequences in H1-depleted chromatin. Histone arrangement in nucleosome core. J Biochem 86: 16591670.

Tagami H, Ray-Gallet D, Almouzni G, Nakatani Y. 2004. Histone H3.1 and H3.3 complexes mediate nucleosome assembly pathways dependent or independent of DNA synthesis. Cell 116: 51-61.

Talbert PB. Masuelli R, Tyagi AP, Comai L, Henikoff S. 2002. Centromeric localization and adaptive evolution of an Arabidopsis histone H3 variant. Plant Cell 14: 1053-1066.

Talbert PB, Bryson TD, Henikoff S. 2004. Adaptive evolution of centromere proteins in plants and animals. J Biol 3: 18.

Talbert PB, Bayes JJ, Henikoff S. 2008. Evolution of centromeres and kinetochores: A two-part fugue. In The kinetochore (ed. P De Wulf and WC Earnshaw), pp. 193-230. Springer, Berlin.

Tomonaga T, Matsushita K, Yamaguchi S, Oohashi T, Shimada H, Ochiai T, Yoda K, Nomura F. 2003. Overexpression and mistargeting of centromere protein-A in human primary colorectal cancer. Cancer Res 63: 3511-3516.

Van Hooser AA, Ouspenski II, Gregson HC, Starr DA, Yen TJ, Goldberg ML, Yokomori K, Earnshaw WC, Sullivan KF, Brinkley BR. 2001. Specification of kinetochore-forming chromatin by the histone H3 variant CENP-A. J Cell Sci 114: 3529-3542.

Vermaak D, Hayden HS, Henikoff S. 2002. Centromere targeting element within the histone fold domain of Cid. Mol Cell Biol 22: 7553-7561.

Visnapuu ML, Greene EC 2009. Single-molecule imaging of DNA curtains reveals intrinsic energy landscapes for nucleosome deposition. Nat Struct Mol Biol 16: 1056-1062.

Wang H, Dalal Y, Henikoff S, Lindsay S. 2008. Single-epitope recognition imaging of native chromatin. Epigenetics Chromatin 1: 10 .

Warburton PE. 2004. Chromosomal dynamics of human neocentromere formation. Chromosome Res 12: 617-626.

Yan H, Talbert PB, Lee HR, Jett J, Henikoff S, Chen F, Jiang J. 2008. Intergenic locations of rice centromeric chromatin. PLoS Biol 6: e286.

Zeitlin SG, Baker NM, Chapados BR, Soutoglou E, Wang JY, Berns MW, Cleveland DW. 2009. Double-strand DNA breaks recruit the centromeric histone CENP-A. Proc Natl Acad Sci 106: $15762-15767$. 


\section{$\$_{\mathrm{CSH}}^{\infty}$ Cold Spring Harbor Symposia SYMPOSIA on Quantitative Biology}

\section{Epigenetic Inheritance of Centromeres}

S. Henikoff and T. Furuyama

Cold Spring Harb Symp Quant Biol 2010 75: 51-60 originally published online November 3, 2010 Access the most recent version at doi:10.1101/sqb.2010.75.001

References This article cites 85 articles, 30 of which can be accessed free at: http://symposium.cshlp.org/content/75/51.full.html\#ref-list-1

License

Email Alerting Receive free email alerts when new articles cite this article - sign up in the box at the Service top right corner of the article or click here.

To subscribe to Cold Spring Harbor Symposia on Quantitative Biology go to:

http://symposium.cshlp.org/subscriptions 